\title{
EFFICACY OF ADMISSION CARDIOTOCOGRAPHY (ADMISSION TEST) TO PREDICT OBSTETRIC OUTCOME
}

\author{
Dwarakanath. L, Lakshmikantha. G, Chaitra S. K,
}
1. Associate Professor, department of Obstetrics \& Gynaecology, Sri Siddhartha Medical College, Tumkur, Karnataka, India
2. Assistant Professor, department of Obstetrics \& Gynaecology, Sri Siddhartha Medical College, Tumkur, Karnataka, India
3. Resident, department of Obstetrics \& Gynaecology, Sri Siddhartha Medical College, Tumkur, Karnataka, India

\section{CORRESPONDING AUTHOR}

Dr. Lakshmikantha G, \# 249, $3^{\text {rd }}$ A main, near Amba Bhavani circle , NE of NR Mohalla, Mysore,

Karnataka, INDIA 570007

E-mail: drlakshmikanthag@yahoo.co.in

Ph: 00919740810611

CAPSULE: Admission test can be used as an effective screening technique to detect pre-existing fetal distress. ABSTRACT: OBJECTIVE: To determine the efficacy of admission test on perinatal outcome and levels of obstetric interventions. METHODS: Prospective study on 200 pregnant women at admission by cardiotocography and assess the perinatal outcome and obstetric interventions. RESULTS: Incidence of reactive trace was $69 \%$, suspicious $24 \%$ and ominous $7 \%$. Out of 200 cases $159(80 \%)$ had vaginal delivery, 28(14\%) had LSCS and 13(6\%) had instrumental delivery. There was increased incidence of operative delivery and NICU admissions in suspicious and ominous tracings. This study has a sensitivity of $76 \%$ and positive predictive value(PPV) of $96 \%$, specificity of $77 \%$ and negative predictive value(NPV) of $33 \%$ for a reactive test. CONCLUSION: Admission test is an effective predictive technique to detect preexisting fetal distress and plan early intervention to prevent adverse perinatal outcome.

KEY WORDS: Cardiotocography, Admission test, APGAR Score, cord pH

INTRODUCTION: Labor poses physiological stress to all fetuses during the transition from intrauterine to extrauterine environment. The fetal distress in labour is a common occurrence and a cause of concern for both the patient and her obstetrician. Therefore every fetus deserves intrapartum fetal monitoring (1). Routine electronic monitoring of the fetal heart rate in labor is becoming an established obstetric practice in the western world. Widely used in both high and low risk obstetrical patients, electronic fetal monitoring is considered to be a reliable and valid technology to measure the fetal heart rate and uterine contractions(2). In labor wards with few monitors, selection of patients for continuous monitoring or intermittent auscultation is necessary. Risk assessment based on antepartum factor is often insufficient for patient selection as intrapartum fetal morbidity and mortality are not uncommon in low risk patients. In such situations a short recording of fetal heart rate and uterine contraction pattern during labor by electronic fetal monitoring device for a period of 20-30 minutes on admission to labor room is regarded as "Admission Test". It was found that admission test could be used as screening procedure to detect pre-existing fetal hypoxia and plan early intervention to prevent adverse perinatal outcome. 
This study evaluates the role of admission test in intrapartum patients to predict fetuses at risk for adverse outcome and levels of obstetric interventions in both high risk and low risk cases.

METHODS: A prospective study carried out on 200 pregnant women admitted to labor room between September 2010 and June 2011. Preterm labor and pregnancy with fetal congenital anomalies were excluded from the study. Admission test tracings are grouped as reactive, suspicious and ominous. Reactive test includes normal baseline FHR of 110-160 beats per minute, variability of 5-25 beats per minute in the absence of decelerations, presence of two accelerations of 15 beats per minute over the baseline lasting for 15 seconds. Suspicious test includes baseline FHR of $<110$ and $>160$ beats per minute, variability of $<5$ beats per minute, absence of acceleration for $>40$ minutes, with transient deceleration. Ominous trace includes baseline FHR of $<90$ beats per minute and $>180$ beats per minute, variability of $<5$ beats per minute for $>90$ minutes, absence of acceleration, presence of persistent decelerations. If the test was reactive, intermittent auscultation was advised for monitoring of the fetus. If the test was suspicious, continuous electronic monitoring was advised and if the test was ominous immediate delivery by the favourable route was planned. Mode of delivery, APGAR score at $1^{\text {st }}$ minute and and $5^{\text {th }}$ minute, NICU admission and cord $\mathrm{pH}$ were evaluated.

RESULTS: In this study 200 pregnant women with both low risk and high risk except preterm and fetal anomalies who were in labor were analyzed.Tabe1 shows the type of admission test with respect to risk of pregnancy. If the admission test to delivery interval exceeded 5 hours there was two fold increase in operative delivery. Indication for forceps after reactive admission test was fetal distress in 2 cases and it was applied prophylactically for maternal cardiac disease in one case. Indication for LSCS after reactive admission test was fetal distress in 4 cases, prolonged labor in 2 cases, cervical dystocia in one case, prolonged PROM in two cases and CPD in one case. In suspicious admission test, out of 48 cases 11 cases (23\%) taken up for LSCS and 1 case (2\%) delivered by ventouse extraction. Fetal distress was the indication in 7 cases $(15 \%)$ of LSCS and 2 cases for obstructed labour and 2 cases for prolonged PROM. There were 4 (8\%) cases of forceps delivery of which one had fetal distress.

In ominous traces out of 14 cases (7\%), 2 cases were delivered normally, forceps was applied for 3 cases and ventouse extraction was done for 2 cases where there was fetal distress in one and outlet contraction in the other. Out of 7 cases delivered by LSCS, fetal distress was the indication in 5 cases, CPD in one case and prolonged PROM in the other.

Umbilical cord $\mathrm{pH}$ was evaluated for all the cases and acidotic babies were detected( table 3).In 189(95\%) cases APGAR score was found satisfactory i.e $>7$ at 1 and 5 minutes. All eleven neonates (5\%) with low APGAR score were admitted to NICU. Three neonates died due to meconium aspiration syndrome and two died due to septicemia secondary to chorioamnionitis.

DISCUSSION: Electronic fetal monitoring (EFM) has been a subject of controversy for the last two decades. Several authors criticized the policy of EFM stating that it will lead to an increase in intervention rates with no evidence of fetal benefits(2). Electronic fetal monitoring can be used as an Admission Test to detect the fetal intrauterine asphyxia already present at admission(3,4). This would help in identifying population of women who would require continuous electronic fetal monitoring or intermittent auscultation. Antepartum risk factors are 
not accurate as predictors of fetal outcome as fetal heart rate changes and fetal acidosis might occur with some frequency in high as well as low risk group. Two hundred pregnant women included in the present study were from high risk as well as low risk group admitted to the labor room in active labor. $40.5 \%$ of the cases were in high risk group and 59.5\% were in low risk group. In Kamal Buckshee study $32 \%$ were in high risk and $68 \%$ were in low risk(5). Both high and low risk cases were included in the Rose Jophy et. al study(6). In Ingermarson study only low risk patients were selected for the study(7). In the present study high risk factors are PIH, PROM, eclampsia, severe anaemia and bad obstetric history. Observation in the present study shows that only 4 cases $(2.8 \%)$ underwent LSCS for fetal distress in the reactive group. In abnormal test 12 cases (19.35\%) had LSCS only for fetal distress during labor.

In cases of reactive admission test out of 138 cases, 125 (90.5\%) had vaginal delivery, $10(7.25 \%)$ had LSCS and only $3(2.17 \%)$ cases had forceps delivery. Out of 3 cases that had forceps delivery one was a cardiac case where prophylactic forceps was used and in 2 cases $(2.7 \%)$ for fetal distress. It is in comparison to Rose Jophy et. al study wherein out of 136 reactive patients $5(3.67 \%)$ had forceps delivery for fetal distress. Ten women underwent LSCS but only $4(2.8 \%)$ were for fetal distress in comparison to Rose Jophy study in which $3(2.2 \%)$ had LSCS for fetal distress in the reactive group. APGAR score less than 7 was seen in 1 case $(0.7 \%)$ as compared to Kamal Buckshee's study where 3 cases (3.5\%) had APGAR less than 7.In Rose Jophy et. al study APGAR score less than 7 was not seen in reactive group. Fetal heart abnormalities like bradycardia were seen in 6 cases (4.3\%). In Rose Jophy study fetal distress was observed in 9 cases (6.6\%) which was correlating with the present study. Normal cord pH was seen in 109 neonates (79\%) and acidosis was seen in 29 neonates (21\%) after a reactive test and admitted to NICU.

Normal tracing early in labor does not give us total assurance that abnormalities will not occur late in labor. When admission test was reactive and the admission test delivery interval has prolonged for more than 5 hours the incidence of fetal distress is doubled, leading to more operative deliveries as compared to reactive test with interval less than or equal to $5 \mathrm{hrs}$. It shows that admission test is not significant in predicting fetal outcome if admission test delivery interval exceeds more than $5 \mathrm{hrs}$.

Suspicious admission test was seen in $48(24 \%)$ cases, 32 cases had vaginal delivery (66.6\%), 11 cases were taken up for LSCS (22.9\%). Vacuum was applied in 1 case $(3.25 \%)$ for fetal distress. Out of 11 LSCS cases 7 cases (29\%) were indicated for fetal distress. Two LSCS were done for prolonged labor and 2 for prolonged PROM. These observations were compared to Rose Jophy study where in 8 cases(14\%)underwent LSCS for fetal distress, in Ingermarson study 5 cases (10\%)were taken for LSCS and in Kamal Buckshee study 3 cases (8\%) were taken for LSCS for fetal distress. Instrumental delivery for foetal distress was accountable to $4.2 \%$ in the suspicious group. Fetal heart abnormalities were seen in 11 cases (23\%) of which fetal tachycardia was seen more often than bradycardia. Meconium stained liquor was seen in 13 cases $(27.1 \%)$ in comparison to Kamal Buckshee case study where one case had meconium stained liquor (9\%).APGAR score less than 7 was seen in 2 cases $(6.4 \%)$ and were admitted to NICU. In Kamal Buckshee study no baby was seen with APGAR less than 7. Cord pH was seen in 22 cases (45.8\%) whereas acidosis was seen in 26 cases(54\%) and admitted to NICU.

An ominous admission test was seen in 14 cases (7\%), in which 2 cases (14\%) were delivered vaginally, 5 cases (35\%) by instrumental (3 forceps, 2 vacuum) and 7 cases (50\%) were taken for LSCS. Out of 7 cases LSCS was done for fetal distress in 5 cases (35\%). In comparison to Kamal Buckshee's study LSCS was done in 2 cases (50\%) for fetal distress. In 
Rose Jophy study LSCS was done for 2 cases in ominous group accounting to $33.33 \%$.In Ingermarson study 2 cases (20\%) were taken for LSCS for fetal distress. Fetal heart rate abnormalities such as bradycardia were seen in cases among which 9 were admitted to NICU for birth asphyxia, meconium aspiration syndrome and also low birth weight. This will account to $64.2 \%$ of all ominous cases. In Rose Jophy study 4 cases had fetal abnormalities accounting to 66.6\%.APGAR score less than 7 was seen in 8 cases (57\%). This was to compare Rose Jophy et al study in which 3 neonates had low APGAR score in ominous group accounting to 50\%. In Kamal Buckshee's study APGAR less than 7 was seen in 1 case (25\%). In present study one mother had jaundice and baby was deeply asphyxiated and could not be resuscitated. The mother with chorioamnionitis was delivered by LSCS but the baby had meconium aspiration syndrome and died due to septicemia. The other patient had viral fever for 4 days before delivery and thick meconium stained liquor was noted. Baby died due to septicemia. Perinatal mortality was $28 \%$ in the ominous group in the present study. Thick meconium stained liquor was seen in 8 cases (57.1\%). Meconium liquor was seen in one case comprising to $16.7 \%$ in Rose Jophy study. In Kamal Buckshee's study there were no cases detected with meconium stained liquor. Thin meconium was seen in $6.8 \%$ of all cases in Ingermarson et. al study where as thick meconium was observed in $2.8 \%$ of patients. In the present study thin meconium liquor was seen in 5 cases comprising to $35.7 \%$. Normal cord $\mathrm{pH}$ seen in 5 cases(36\%). Acidosis either mild or severe form was observed in 9 cases (64\%) and 4 infants died in this group in NICU.

CONCLUSION: Admission Test is a simple economic tool and easy to perform. Admission test has a good predictive value for fetal well being for next few hours of labor. It is a valuable screening test to identify the fetuses at risk for non reassuring fetal status. It is found that admission test can be used as a screening procedure to detect pre-existing fetal hypoxia and plan early intervention to prevent adverse perinatal outcomes.

\section{REFERENCES :}

1. Veconica Ilene yuel, VaneetKaur. J Obs and Gynaec today, 2005; VolX, No.8.

2. C Woods,P.Renou,J.Oat."A controlled trial of fetal heart rate monitoring in a low risk obstetric population". AmJObstet and Gynecol.1981:141:527.

3. Ros Goddard et al. Electronic fetal monitoring. BMJ 2001; 1322:1436-1437.

4. Das V, Katiyar N, Malik GK. Role of admission test. J ObstetGynecol India.2001;51(1):48-50.

5. Buckshee Kamal, DekaDeepika, Padmaja V., et. al. "Admission test as predictor of fetaloutcome". J Obstet and Gynaec of India, 1999; 49(2): 36-37.

6. Rose Jophy ,Annamma Thomas, PrabhaJairaj. J obstet and gynae of India Vol: 52,No. 5; September/October 2002: 26-29.

7. IngermarsonI et al. "Admission test: Screening test for fetal distress in labor". ObstetGynecol, 1986: $68:$ 800-806. 


\section{TABLES:}

\begin{tabular}{|l|c|c|c|}
\hline Type of CTG & Number & High risk & Low risk \\
\hline Reactive & 138 & $\begin{array}{c}45 \\
(53.1 \%)\end{array}$ & $\begin{array}{c}95 \\
(79.8 \%)\end{array}$ \\
\hline Suspicious & 48 & $\begin{array}{c}29 \\
(35.8 \%)\end{array}$ & $\begin{array}{c}19 \\
(15.9 \%)\end{array}$ \\
\hline Omnious & 14 & 9 & 5 \\
$(11.1 \%)$ & $(4.2 \%)$ \\
\hline Total & 200 & 81 & 119 \\
\hline
\end{tabular}

$P$ value $:<0.01$

TABLE 1: Admission test v/s Risk group.

\begin{tabular}{|l|c|c|c|c|}
\hline \multirow{2}{*}{$\begin{array}{l}\text { Type of CTG (OUT OF } \\
200 \\
\end{array}$} & \multicolumn{4}{|l|}{ cases) } \\
\cline { 2 - 5 } & Vaginal & Forceps & Ventouse & LSCS \\
\hline REACTIVE (138) & 125 & 3 & 0 & 10 \\
& $(90.5 \%)$ & $(2.2 \%)$ & & $(7.2 \%)$ \\
\hline SUSPICIOUS (48) & 32 & 4 & 1 & 11 \\
& $(66.6 \%)$ & $(8.3 \%)$ & $(2.1 \%)$ & $(22.9 \%)$ \\
\hline OMINOUS (14) & 2 & 3 & 2 & 7 \\
& $(14.3 \%)$ & $(21.4 \%)$ & $(14.3 \%)$ & $(50 \%)$ \\
\hline TOTAL & 159 & 10 & 3 & 28 \\
& $(79.5 \%)$ & $(5 \%)$ & $(1.5 \%)$ & $(14 \%)$ \\
\hline
\end{tabular}

TABLE 2: Admission Test v/s Mode of Delivery

\begin{tabular}{|c|c|c|c|}
\hline Type of CTG & Number & Normal cord Ph & Acidosis \\
\hline Reactive & 138 & 109 & 29 \\
& & $(78.9 \%)$ & $(21 \%)$ \\
\hline Suspicious & 48 & 22 & 26 \\
& & $(45.8 \%)$ & $(54.1 \%)$ \\
\hline Ominous & 14 & 5 & 9 \\
& & $(35.8 \%)$ & $(64.2 \%)$ \\
\hline Total & 200 & 137 & 63 \\
& & $(68.5 \%)$ & $(31.5 \%)$ \\
\hline
\end{tabular}

$P$ value $:<0.01$

TABLE 3: Admission test v/s Cord pH. 


\begin{tabular}{|c|c|c|c|c|c|c|}
\hline Type of Admission & Rose & y et. Al & Prese & study & Ingern & al study \\
\hline Reactive & 136 & $68 \%$ & 138 & $69 \%$ & 982 & $94.3 \%$ \\
\hline Fetal distress(FD) & 9 & $6.6 \%$ & 6 & $4.3 \%$ & 32 & $1.3 \%$ \\
\hline & 127 & $93.3 \%$ & 132 & $95.7 \%$ & 950 & $98.7 \%$ \\
\hline Suspicious & 58 & $29 \%$ & 48 & $24 \%$ & 49 & $4.7 \%$ \\
\hline FD +ve & 16 & $27.6 \%$ & 11 & $22.9 \%$ & 5 & $10.2 \%$ \\
\hline FD -ve & 42 & $72.4 \%$ & 37 & $77.1 \%$ & 44 & $89.8 \%$ \\
\hline Ominous & 6 & $15.5 \%$ & 14 & $7 \%$ & 10 & $1 \%$ \\
\hline FD +ve & 4 & $66.6 \%$ & 9 & $64.3 \%$ & 4 & $40 \%$ \\
\hline FD -ve & 2 & 3230 & 5 & 3570 & 6 & $600 \%$ \\
\hline & 2 & $33.3 \%$ & $\mathbf{5}$ & $35.7 \%$ & 6 & $60 \%$ \\
\hline
\end{tabular}

TABLE 4: Acute fetal distress following admission test.

\begin{tabular}{|l|c|c|l|c|}
\hline STUDY & Sensitivity & Specificity & PPV & NPV \\
\hline Kamal buckshee et. al(5) & $87.5 \%$ & $27.7 \%$ & $84.7 \%$ & $33.3 \%$ \\
\hline Ingermarson et.al(7) & $95.48 \%$ & $31.1 \%$ & $96.8 \%$ & $23.7 \%$ \\
\hline Rose Jophy et.al(6) & $30.9 \%$ & $98.45 \%$ & $93.4 \%$ & $66.6 \%$ \\
\hline Present study & $\mathbf{7 5 . 8 \%}$ & $\mathbf{7 6 . 9 \%}$ & $\mathbf{9 5 . 6 \%}$ & $\mathbf{3 2 . 2 5 \%}$ \\
\hline
\end{tabular}

Table 5: Overall efficacy of admission test. 